\title{
Measurement of ion temperature and density profiles with a time-of-flight type neutral-particle analyzer
}

\author{
M. Ichimura, ${ }^{a)}$ H. Higaki, T. Kawabata, D. Inoue, H. Nagai, S. Kakimoto, Y. Yamaguchi, \\ K. Horinouchi, K. Ide, K. Nakagome, and T. Cho \\ Plasma Research Center, University of Tsukuba, Tsukuba, Ibaraki 305-8577, Japan
}

(Presented on 19 April 2004; published 5 October 2004)

\begin{abstract}
High beta plasmas in the anchor cell of the GAMMA 10 tandem mirror are formed by using a fundamental ion cyclotron resonance heating. Because the closed resonance surface exists near the midplane of the anchor cell, strongly peaked profile of the plasma pressure will be formed. To measure the axial profiles of the ion temperature and density, a time-of-flight type neutral-particle analyzer (TOF-NPA) has been developed. TOF-NPA can detect neutral particles and photons separately at the same time. The ion temperature and density have been evaluated from the particle and photon signals, respectively. The peaked profile of the ion temperature within the closed resonance surface has been confirmed.
\end{abstract}

(C) 2004 American Institute of Physics. [DOI: 10.1063/1.1785265]

\section{INTRODUCTION}

When discussing macro- and microstability in the mirror devices it is important to know the plasma pressure distribution along the magnetic field line. An ion temperature of more than $10 \mathrm{keV}$ has been performed with the ion cyclotron range of frequency (ICRF) heating in the central cell of the GAMMA 10 tandem mirror. ${ }^{1}$ Because the cyclotron resonance layer exists near the midplane of the central cell, plasmas with a strong pressure anisotropy (more than 10), which is defined as a ratio of perpendicular to parallel pressure to the magnetic field line, have been produced. A microinstability, for example, Alfvén ion cyclotron mode is spontaneously excited in such plasmas with the strong pressure anisotropy. ${ }^{2}$ In magnetohydrodynamics (MHD) instability, for example, a flute interchange mode depends on the critical beta ratio of the central to anchor cells, which is related to the pressure weighting on the field line with a good or bad curvature, that is, pressure profile in both cells. In this article, the development of a time-of-flight type neutral-particle analyzer (TOFNPA) installed for evaluation of ion temperature and density profiles of the anchor cell is described.

\section{EXPERIMENTAL SETUP}

GAMMA 10 is a minimum- $B$ anchored tandem mirror with axisymmetric plug/barrier cells at both ends. The central cell is $5.6 \mathrm{~m}$ long with the field strength of $0.4 \mathrm{~T}$ at the midplane. Plasmas are started and sustained by applying ICRF pulse (RF1), of which frequencies are $9.9 \mathrm{MHz}$ (east) and $10.3 \mathrm{MHz}$ (west), with short pulse (1 ms) plasma guns from both ends and gas puffings. So-called Nagoya type-III antennas, which are installed near both ends of the central cell, are used for RF1 antennas. To avoid the strong interference, the frequency of the west antenna is slightly higher than the frequency of the east antenna. In the startup scenario

${ }^{a)}$ Electronic mail: ichimura@prc.tsukuba.ac.jp on GAMMA 10, one of the most important conditions is the ion heating of anchor plasmas to keep MHD stability of the whole GAMMA 10 plasma. $^{3}$ The frequencies of RF1 are selected as the fundamental cyclotron resonance exists near the midplane of the anchor cell. The field strength is $0.6 \mathrm{~T}$ at the midplane and the mirror ratio is 3.3. Fast Alfvén waves excited in the central cell propagate to the anchor cell. At the inner transition region between both cells, a part of the fast waves is converted to slow waves and heats ions in the anchor cell. ${ }^{4,5}$ It should be noted that the cyclotron resonance surface in the minimum- $B$ field configuration is closed to the center.

Figure 1 shows a schematic drawing of TOF-NPA, which consists of a chopper disk, a flight tube, and a particle detector. Hydrogen neutral atoms and photons are emitted from plasmas perpendicularly and reach to the chopper disk, which is located $3 \mathrm{~m}$ from the plasma axis. There are 24 slits of $0.2 \mathrm{~mm}$ width, which determine the energy resolution, and $1 \mathrm{~cm}$ height at a radius of $75 \mathrm{~mm}$ on the chopper disk. The rotation frequency of the disk is about $30000 \mathrm{rpm}$. In front of the disk, there is a fixed slit of $0.2 \mathrm{~mm}$ width. The detector can see the plasma at every $100 \mu \mathrm{s}$. The chopped neutral atoms and photons travel $3.2 \mathrm{~m}$ in the flight tube and impinge on a copper target of the particle detector, which is biased at $-20 \mathrm{kV}$. Secondary electrons ejected from the target are accelerated onto an aluminized and grounded plastic scintillator by the bias voltage. Finally, the electric signal is obtained as an output of a photomultiplier. By pivoting TOFNPA against the magnetic field line, parameters in the axial direction can be measured.

Figure 2 shows typical time-of-flight spectra detected at three different positions in the axial direction. There are large peaks of photons at $t=0$. The following particle signals give the information of the energy distribution of anchor ions. The coefficient of the secondary electron emission has the energy dependence. ${ }^{6}$ There are several reports on the coefficient. The error was said to be due mostly to the technique of 


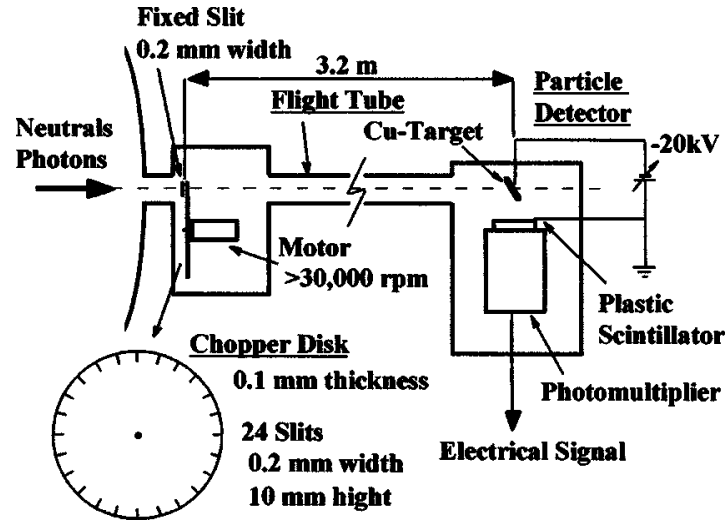

FIG. 1. Schematic drawing of a TOF-NPA.

measuring rather than to the emitter material used. This is attributed to the influence of contamination on material surfaces. It is predicted in Ref. 7 that the data obtained by different investigators agrees to within $10 \%$.

Because of the high efficiency for detecting low energy neutrals, TOF-NPA is considered to be useful to measure neutrals of whose energy is a few hundred $\mathrm{eV}$ and below. Plasmas with ion temperature in the $\mathrm{keV}$ range are produced in the anchor cell of GAMMA 10. For example, the time-offlight of the neutral hydrogen atom with energy of $5 \mathrm{keV}$ is about $5 \mu \mathrm{s}$ in the present experiment. Because the difference between TOF spectra from the midplane and off midplane of the anchor cell is clear in Fig. 2, it is still useful to evaluate the change of the energy distribution relatively. The energy distribution is obtained every $100 \mu \mathrm{s}$. To obtain a smooth energy spectrum, summing up more than ten slits is needed. The time resolution is determined from the averaging process.

The photon signal becomes good indicator of the electron density. The energy spectrum of photons has been deduced from the measurements using several filters, each of which has a different energy transmission band. ${ }^{8}$ It has been confirmed that the photon signals are mainly attributed to the line emissions due to light impurities. In Fig. 3, peak values of the photon signal are plotted as a function of the line density. It is clearly shown that the photon signal indicates

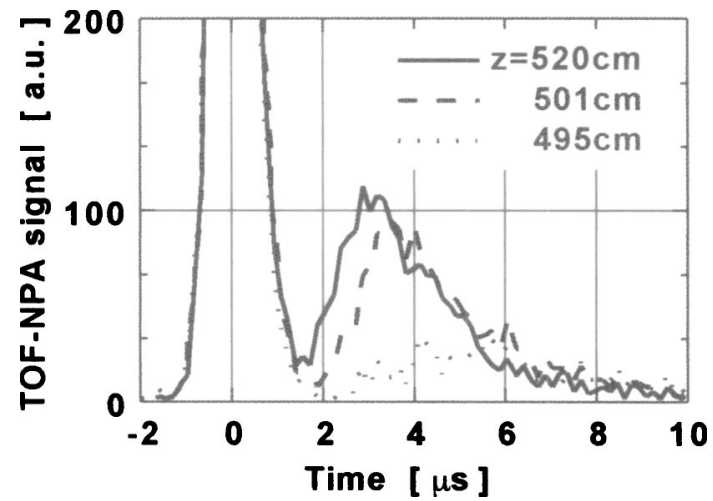

FIG. 2. Typical time-of-flight spectra from three different axial locations. Distances from the midplane of the central cell are indicated.

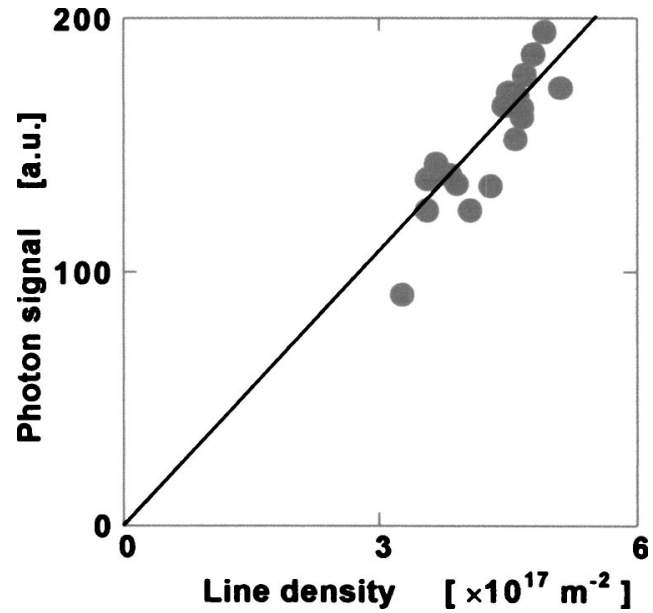

FIG. 3. Amplitude of the photon signal vs line density at the midplane of the anchor cell.

the relative density. Assuming the same radial profile, the density distribution in the axial direction is estimated from the distribution of the photon signal.

\section{EXPERIMENTAL RESULTS AND DISCUSSION}

The ion temperature near the midplane of the anchor cell can be estimated from the diamagnetism and the line density measurements. In Fig. 4, ion temperatures deduced from TOF-NPA are plotted as a function of the value of the diamagnetic signal divided by the line density. The linear correlation is confirmed in $\mathrm{keV}$ range plasmas.

As clearly shown in Fig. 2, the TOF spectrum from the off midplane of the anchor cell shows lower temperature than that from the midplane. Figure 5 shows the axial distribution of the ion temperatures deduced from the TOF signals. The axial profile of the magnetic field strength and the location of the ion cyclotron resonance are indicated. It is clearly shown that the hot ions are confined in the resonant sphere, which is surrounded by the equimagnetic field strength surface. The ion temperature is almost flat near the midplane and decreases abruptly at the resonant surface. The minimum- $B$ configuration is broken outside the location in-

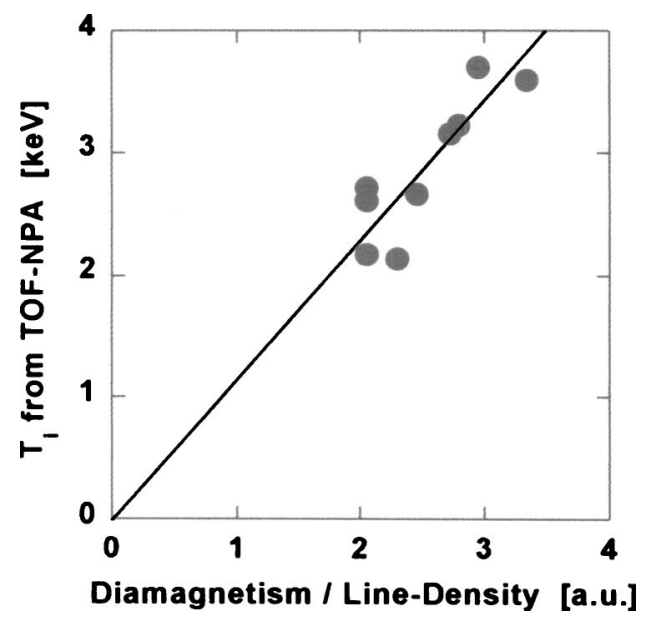

FIG. 4. Ion temperatures deduced from TOF-NPA vs values of diamagnetism divided by line density at the midplane of the anchor cell. 


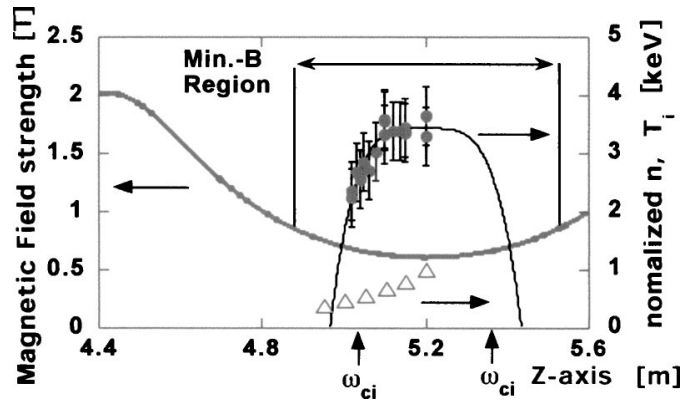

FIG. 5. Axial profile of $T_{i}$ and density deduced from TOF-NPA. Density is normalized at the anchor midplane $(z=5.2 \mathrm{~m})$. Magnetic field strength and locations of ion cyclotron resonance and minimum- $B$ configuration are indicated.

dicated in Fig. 5, that is; the surface with the equimagnetic field strength begins to open. The hot ion plasmas are deeply confined within the minimum- $B$ configuration. The axial distribution of the density deduced from the photon signals is also plotted in Fig. 5. The density decreases gradually along the magnetic field line. The perpendicular pressure distribu- tion can be evaluated from ion temperature and density profiles obtained along the magnetic field line. Then, the parallel pressure is calculated from the measured perpendicular pressure and the magnetic field configuration. ${ }^{9}$

The anisotropy, the macrostability in GAMMA 10, and the microinstability induced from the anisotropy will be discussed in other articles.

\section{ACKNOWLEDGMENT}

The authors acknowledge the GAMMA 10 group at the University of Tsukuba for their collaboration.

${ }^{1}$ T. Tamano et al., Proceedings of 15 th International Conference on Plasma Physics and Controlled Nuclear Fusion Research, Seville, 1994, (IAEA, Vienna, 1995), Vol. 2, p. 399.

${ }^{2}$ M. Ichimura et al., Phys. Rev. Lett. 70, 2734 (1993).

${ }^{3}$ M. Ichimura et al., Nucl. Fusion 28, 799 (1988).

${ }^{4}$ M. Inutake et al., Phys. Rev. Lett. 65, 339 (1990).

${ }^{5}$ H. Hojo et al., Phys. Rev. Lett. 66, 1866 (1991).

${ }^{6}$ C. F. Barnett and J. A. Ray, Nucl. Fusion 12, 65 (1972).

${ }^{7}$ A. I. Kislyakov et al., Sov. Phys. Tech. Phys. 20, 986 (1975).

${ }^{8}$ F. Tsuboi et al., Rev. Sci. Instrum. 60, 2868 (1989).

${ }^{9}$ J. B. Taylor, Am. J. Phys. 26, 529 (1963). 